\title{
VANTAGEM COMPETITIVA: O CONSTRUTO E A MÉTRICA
}

RESUMO

Apesar de quase onipresente nos trabalhos de estratégia e áreas correlatas, o conceito de vantagem competitiva continua sendo foco de debate na academia por não possuir uma definição aceita por consenso. Uma manifestação disso é a recente discussão quanto aos seus fundamentos lógicos e filosóficos, e o questionamento de sua própria existência. O presente trabalho oferece uma proposta de definição constitutiva e operacional de vantagem competitiva que possibilita o desenvolvimento de uma métrica. Essa métrica é aplicada em um trabalho empírico que mede a vantagem competitiva de 2.805 empresas em 16 países, com um total de 13.259 observações. O trabalho demonstra que a existência da vantagem competitiva não é um fato raro, e que cerca de 10\% a 12\% das empresas analisadas apresentam uma vantagem competitiva estatisticamente significativa, enquanto que uma porcentagem similar apresenta uma desvantagem competitiva igualmente significativa.

\section{Flávio Carvalho de Vasconcelos \\ FGV-EAESP}

\section{Luiz Artur Ledur Brito FGV-EAESP}

\begin{abstract}
Despite ubiquitous in strategy and related fields, the concept of competitive advantage continues to be under debate in the academy still lacking a precise and widely accepted definition. A manifestation of this situation is the current debate about its philosophical grounds, and the questioning of its own existence. This paper offers a constitutive definition that allows an operationalization of the concept and the development of metric for the competitive advantage. This metric is applied to a sample of 2,805 firms in 16 countries, with a total of 13,259 observations. The analysis indicates the occurrence of competitive advantage in 10\% to $12 \%$ of the firms with a similar percentage showing a competitive disadvantage. It demonstrates that the occurrence of competitive advantage is not a rare event. A statistical test is proposed to verify its statistical significance.
\end{abstract}

PALAVRAS-CHAVE Vantagem competitiva, estratégia, competição, recursos, NAFTA, Europa.

KEY WORDS Strategy, competitive advantage, resources, competition, NAFTA, Europe. 


\section{INTRODUÇÃo}

Como as empresas atuam? Por que as empresas são diferentes? Qual a função da central corporativa nas organizações multidivisionais? O que determina o sucesso e o fracasso na competição internacional? Essas quatro questões foram selecionadas como os temas fundamentais que definem o campo de estratégia na obra editada por Rumelt, Schendel e Teece (1994), Fundamental Issues in Strategy: a research agenda. A idéia de vantagem competitiva está relacionada a todas essas questões. A vantagem competitiva pode ser vista como o objetivo das ações da empresa, pode ser usada para explicar a diversidade entre as empresas, pode ser vista como o objetivo final da função corporativa e, finalmente, pode explicar o sucesso ou fracasso na competição internacional.

A noção de vantagem competitiva tem suas raízes na prática gerencial e de consultoria (South, 1981), de forma similar à estratégia empresarial (Rumelt, Schendel e Teece, 1991). Com a evolução da estratégia como disciplina acadêmica, a temática da vantagem competitiva ganhou uma abordagem mais científica e formal, deixando de ser uma referência ocasional para se tornar um dos conceitos-chave da disciplina. A visão baseada em recursos (Resource Based View - RBV) oferece um arcabouço próprio para o estudo da vantagem competitiva ao enfatizar que os recursos específicos da empresa são determinantes para a geração de lucro econômico (Barney, 1986a, 1991; Dierickx e Cool, 1989; Peteraf, 1993). Já as teorias de posicionamento estratégico sustentam que a vantagem competitiva pode derivar não apenas de recursos específicos, mas também de posições de mercado privilegiadas (Porter, 1979; Caves, 1984).

Embora o uso da expressão "vantagem competitiva" seja freqüente e comum na literatura de estratégia e de Administração em geral, sua definição não é precisa. $\mathrm{O}$ conceito está, sem dúvida, ligado a um desempenho superior das empresas, mas essa ligação tem múltiplas abordagens, dependendo dos autores e do contexto. Não há consenso, por exemplo, quanto a uma questão fundamental: a vantagem competitiva deve ser tomada como uma causa do desempenho superior ou como a constatação efetiva desse desempenho? Hitt, Ireland e Hoskisson (1999) e Besanko et al. (2004, p. 360) adotam esta última perspectiva quando definem vantagem competitiva como o fato de uma empresa ter um desempenho em termos de lucro econômico superior ao de outras empresas atuantes no mesmo mercado. Já a maior parte dos autores da RBV têm uma abordagem diferente. Barney (1991, p. 102), por exemplo, afirma que uma empresa tem uma vantagem competitiva quando implementa uma estratégia de criação de valor que não é implementada, simultaneamente, por nenhum dos seus concorrentes atuais ou potenciais. O desempenho superior seria uma consequência, um resultado provável dessa estratégia, influenciado também pelos custos em adquirir os recursos necessários (Barney, 1986a).

O presente trabalho pretende contribuir para o debate oferecendo uma definição precisa do conceito de vantagem competitiva, inspirando-se, para tanto, em estudos de componentes de variância do desempenho da empresa, em especial no trabalho de Rumelt (1991). O objetivo deste trabalho é oferecer subsídios para o desenvolvimento de definições operacionais específicas sobre o que constitui vantagem competitiva e sobre o modo de mensurá-la.

O restante do trabalho está estruturado da seguinte forma: na próxima seção é apresentado um histórico do surgimento do conceito de vantagem competitiva em estratégia, culminando com o desenvolvimento teórico do conceito na visão baseada em recursos (RBV); a proposta de uma definição constitutiva é então apresentada, seguida do detalhamento do método estatístico empregado e dos resultados obtidos; finalmente, as conclusões e as sugestões para os estudos futuros são apresentadas e comentadas.

\section{ORIGENS HISTÓRICAS DO CONCEITO DE VANTAGEM COMPETITIVA}

Uma das primeiras referências à expressão "vantagem competitiva" na literatura de estratégia pode ser encontrada em Ansoff (1965, p. 188-194), que a definiu como a vantagem de perceber, de forma pró-ativa, tendências de mercado à frente dos concorrentes e de ajustar a oferta em função dessa antecipação. Por exemplo, quando a General Motors, na década de 1920, percebeu a demanda potencial no mercado norte-americano para meios de transporte pessoais mais luxuosos, isso lhe permitiu obter uma importante vantagem em relação à Ford (líder na produção de veículos de baixo custo com seu modelo $\mathrm{T}$ ), invertendo a situação competitiva das duas empresas. Mas nem todas as tentativas de prever as tendências do mercado foram bem sucedidas. O Ford Edsel e a tentativa antecipada da RCA de comercializar a TV colorida são exemplos de antecipações prematuras.

Há semelhanças entre essa concepção de vantagem competitiva como antecipação de oportunidades e a definição que lhe é dada hoje, embora represente uma abor- 
dagem muito mais próxima do que chamamos agora de "vantagens de pioneiro" ou first-mover advantages, com um papel secundário no arcabouço geral do planejamento estratégico racional. No trabalho posterior de Ansoff (1979), a expressão "vantagem competitiva" desaparece por completo.

A estratégia, em seu período inicial de amadurecimento, estava focada na função de planejar e mobilizar a empresa para seu processo de execução. Andrews (1971) define as quatro funções da estratégia corporativa como: 1) a definição dos objetivos estratégicos, já que apenas a dimensão financeira, como a maximização do lucro, seria inadequada; 2) o planejamento do futuro mais distante que seria necessário para empreendimentos de ciclo mais longo; 3) a capacidade de influenciar o ambiente além de simplesmente responder às suas mudanças; e 4) a criação de objetivos visíveis que mobilizassem e inspirassem a cooperação e o esforço organizacional. A conquista e a manutenção de vantagem competitiva simplesmente não aparecem nos textos mais clássicos de estratégia empresarial, ao contrário do que vai acontecer, com grande freqüência, nos textos contemporâneos.

No final dos anos 70, o tema "competição" começa a aparecer em textos de autores ligados à prática empresarial que, nessa época, capitaneavam o ensino de estratégia ou política de negócios. Nesse mesmo período, as empresas americanas experimentavam um mal-estar com a crescente competição de indústrias estrangeiras, em especial japonesas, que competiam em bases diferentes, com grande sucesso em muitas áreas. Alguns analistas atribuíram esse sucesso ao fato de os japoneses atribuírem um valor estratégico diferente às operações (Hayes e Wheelwright, 1984; Wheelwright, 1981). O resultado desse aumento de competição foi a intensificação da preocupação da indústria norte-americana com o foco competitivo, muito mais do que costumava fazer antes.

Allen (1978), então vice-presidente de estratégia corporativa da General Electric, escreve um artigo na McKinsey Quarterly sobre a necessidade do planejamento estratégico com foco competitivo, descrevendo como a GE vinha fazendo isso, com sucesso, durante a década de 70. A expressão "vantagem competitiva" é usada em vários outros artigos desse mesmo periódico em uma acepção mais ampla, envolvendo unidades de negócios inteiras e não apenas um produto (Ohmae, 1978; Morrison e Lee, 1979). Gluck, Kaufman e Walleck (1980), todos consultores da McKinsey, escrevem um artigo na Harvard Business Review com o título "Strategic management for competitive advantage". No mesmo ano, South (1981), então diretor de planejamento corporati- vo da Clark Equipment Company, publica no Journal of Business Strategy um artigo intitulado "Competitive advantage: the cornerstone of strategic thinking". Nesse momento, final dos anos 1970 e início da década de 1980, a vantagem competitiva começa a ocupar um papel central no campo da estratégia. O planejamento e a liderança passam a ser mecanismos para alcançá-la. O processo de gestão estratégica torna-se gestão da vantagem competitiva, ou seja, o processo de criar, desenvolver e manter vantagens competitivas (South, 1981).

Durante a década de 1980, a abordagem da vantagem competitiva se torna cada vez mais popular. Rothschild (1984a, 1984b), então vice-presidente corporativo de desenvolvimento de negócios e planejamento da General Electric, lança o livro How to gain (and maintain) the competitive advantage in business colocando a vantagem competitiva no centro da estratégia e consolidando a visão prática desenvolvida no final da década de 1970 .

A academia também passa a adotar o conceito. Aaker (1984), por exemplo, propõe que a escolha de uma estratégia de negócios possui dois elementos centrais. O primeiro é a decisão sobre onde competir: a decisão produto-mercado. O segundo é o desenvolvimento de uma vantagem competitiva sustentável. Spence (1984) analisa a criação de vantagem competitiva em empresas multinacionais a partir de políticas de subsídio e restrição de acesso. Caves (1984) interpreta a noção de vantagem competitiva sob o ângulo da organização industrial. Finalmente, Porter (1985) lança o seu segundo livro, com o título Competitive Advantage. Enquanto que na obra original de Porter (1980) o conceito de vantagem competitiva simplesmente não aparece de forma destacada, em 1985 ele vem para a posição central. O objetivo e a medida de sucesso da estratégia passam a ser a conquista de uma vantagem competitiva. O conceito de cadeia de valor é usado por Porter (1985) como ferramenta para explicar a geração da vantagem competitiva. Uma empresa conquistaria uma vantagem competitiva executando as atividades estrategicamente mais importantes da cadeia de valor de forma mais barata ou melhor do que a concorrência.

Elaborando a definição dada por Porter, Ghemawat (1986) propõe que as fontes estruturais de vantagem competitiva podem ser encontradas em fatores ligados à inovação do produto, a processos de produção ou às capacidades de marketing das empresas. Ghemawat (1986) detalha essas categorias genéricas em outras mais específicas. Vantagens competitivas podem decorrer de: 1) benefícios de tamanho (economias de escala, escopo ou curvas de experiência); 2) vantagens de acesso privile- 
giado a recursos (como know-how, matérias-primas, mercados); e 3) do exercício de opções que garantam flexibilidade estratégica.

\section{VANTAGEM COMPETITIVA E 0 CONCEITO DE RBV}

Foi o surgimento da visão baseada em recursos (RBV), durante a década de 1980, que permitiu um tratamento teórico mais técnico ao conceito de vantagem competitiva. Embora não se encontrasse, na formulação inicial de Wernerfelt (1984), a noção de vantagem competitiva, ela não tardaria a aparecer, como de fato aconteceu em uma das obras iniciais de Barney (1986b), onde se abordava a questão da cultura organizacional. Nela, Barney (1986b) procurava examinar a relação existente entre cultura organizacional e desempenho financeiro superior. Para que a cultura contribuísse com tal desempenho, seria necessário que fosse capaz de criar valor econômico, que fosse rara e, finalmente, que fosse de difícil imitação. Nessas condições, a cultura era definida como um componente da vantagem competitiva da empresa. Empresas com uma cultura forte que satisfizesse os três requisitos acima mencionados teriam melhores resultados, em média, do que empresas que não atendessem a esses requisitos.

Continuando essa linha de raciocínio, Barney (1991) define o que se tornará o argumento central da teoria dos recursos em termos de vantagem competitiva, o que, neste artigo, denominamos vantagem competitiva componente:

Diz-se que uma empresa possui uma vantagem competitiva quando está implementando uma estratégia de criação de valor que não esteja sendo simultaneamente implementada por seus concorrentes ou concorrentes potenciais, os quais são incapazes de repetir os benefícios dessa estratégia. (Barney, 1991, p. 102).

Esse tratamento de "vantagens competitivas componentes" não é predominante no campo da estratégia e não aparece claramente na maioria dos textos. Vários autores empregam a expressão "vantagem competitiva" não com o intuito de isolar um fator ou recurso único que contribua para o desempenho da empresa, mas no sentido de se referir ao diferencial de desempenho por ela obtido em seu contexto competitivo. Besanko et al. (2004) e Hitt, Ireland e Hoskisson (1999) definem vantagem competitiva em termos do resultado da empresa no emprego combinado de seus recursos:
Quando uma empresa (ou unidade de negócio dentro de uma empresa com múltiplas unidades de negócio) aufere uma taxa maior de lucro do que a taxa média de lucro de outras empresas disputando o mesmo mercado, a empresa possui uma vantagem competitiva nesse mercado. (Besanko et al., 2004, p. 360).

Essa definição corresponde ao que chamamos, neste artigo, de vantagem competitiva resultante (no singular); ou seja, o resultado dos efeitos combinados dos diversos fatores específicos à empresa que afetam seu desempenho. Prahalad e Hamel (1990) descrevem como "competências centrais" aquelas competências que envolvem aprendizagem coletiva e que podem estar na base de vantagens competitivas. Em uma contribuição teórica mais sistemática, Peteraf (1993) tenta vincular as noções de rendas econômicas à noção de vantagem competitiva no quadro conceitual da teoria dos recursos. O modelo desenvolvido por Peteraf indica que a vantagem competitiva deriva da heterogeneidade de recursos das empresas e da mobilidade imperfeita dos recursos entre elas, reforçados por limites ex ante e ex post a competição.

\section{UMA PROPOSTA DE DEFINIÇÃO CONSTITUTIVA DE VANTAGEM COMPETITIVA}

A idéia de vantagem competitiva está, sem dúvida, ligada ao caráter variável do desempenho das empresas. Assim, uma linha de pesquisa bastante difundida em estratégia é aquela que se baseia na análise dos componentes de variância do desempenho, iniciada por Schmalensee (1985) e desenvolvida por Rumelt (1991), Roquebert, Phillips e Westphall (1996), McGahan e Porter (1997), Hawawini, Subramanian e Verdin (2003), entre outros.

A técnica de componentes de variância decompõe a dispersão do desempenho associando-o a grupos de fatores comuns. O desempenho individual de uma empresa, dentro de uma população de outras empresas, pode ser concebido como influenciado por vários grupos de fatores. A análise de componentes de variância permite avaliar qual percentual da variância total pode ser atribuído a cada grupo de fatores. Por exemplo, é possível determinar o percentual da variância total atribuível à estrutura da indústria (ramo de negócios), às variações transitórias associadas ao tempo, a empresas específicas ou à combinação de todos esses fatores. A Figura 1 ilustra essa concepção de composição de desempenho. 
O conjunto de estudos sobre componentes de variância do desempenho apresenta resultados razoavelmente consistentes e indica que fatores associados a empresas específicas são preponderantes na explicação da heterogeneidade do desempenho observado. Em termos gerais, cerca de 35\% a 50\% da variância observada no desempenho de uma empresa podem ser atribuídos a fatores específicos e peculiares dessa empresa. O ramo de negócios ou setor industrial responde por um percentual menor, que, em termos aproximados, pode representar algo entre $10 \%$ e $20 \%$ da variância total.

Um dos pontos de consenso sobre a noção de vantagem competitiva refere-se à constatação de que ela é específica a cada empresa. Assim, efeitos que resultam de fatores que afetem várias empresas, por exemplo, todas as empresas de um determinado ramo de negócios, ou de determinado ano, não poderiam ser considerados como vantagem competitiva.

A proposta de definição constitutiva da vantagem competitiva deste artigo foi inspirada nessa concepção de vantagem competitiva como algo específico a cada empresa. Embora essa proposta não tenha sido feita anteriormente de maneira formal, Rumelt, Schendel e Teece (1994, p. 43) e McGahan (1999, p. 378) fazem comentários que a sugerem. Este artigo porpõe que a vantagem competitiva seja concebida como a influência líquida de todos os fatores idiossincráticos da empresa sobre o seu desempenho durante um período determinado, excluídas as influências de outros fatores, como a indústria, os fatores temporais e o erro estatístico. Podemos operacionalizar a definição de vantagem competitiva respondendo se ela é uma componente ou resultante e se é temporária ou sustentável.

\section{Componente ou resultante?}

A definição aqui proposta esclarece os conceitos de vantagem competitiva resultante, que seria a influência líquida de todos os fatores idiossincráticos da empresa que afetam seu desempenho, e o de vantagens competitivas componentes. Os conceitos seriam análogos aos de forças componentes e força resultante na física. O fenômeno final mensurável é a aceleração causada pela força resultante. As forças componentes podem ser idealizadas de diferentes formas, bem como seu impacto potencial na aceleração final. Poderíamos, de acordo com essa analogia, idealizar vantagens competitivas componentes como fatores isolados associados de forma única à empresa e que influenciam em seu próprio desempenho. Alguns desses fatores poderiam ter uma influência negativa, ou piorar o desempenho. Eles constituiriam uma

Figura 1 - Vários grupos de fatores que determinam o desempenho.

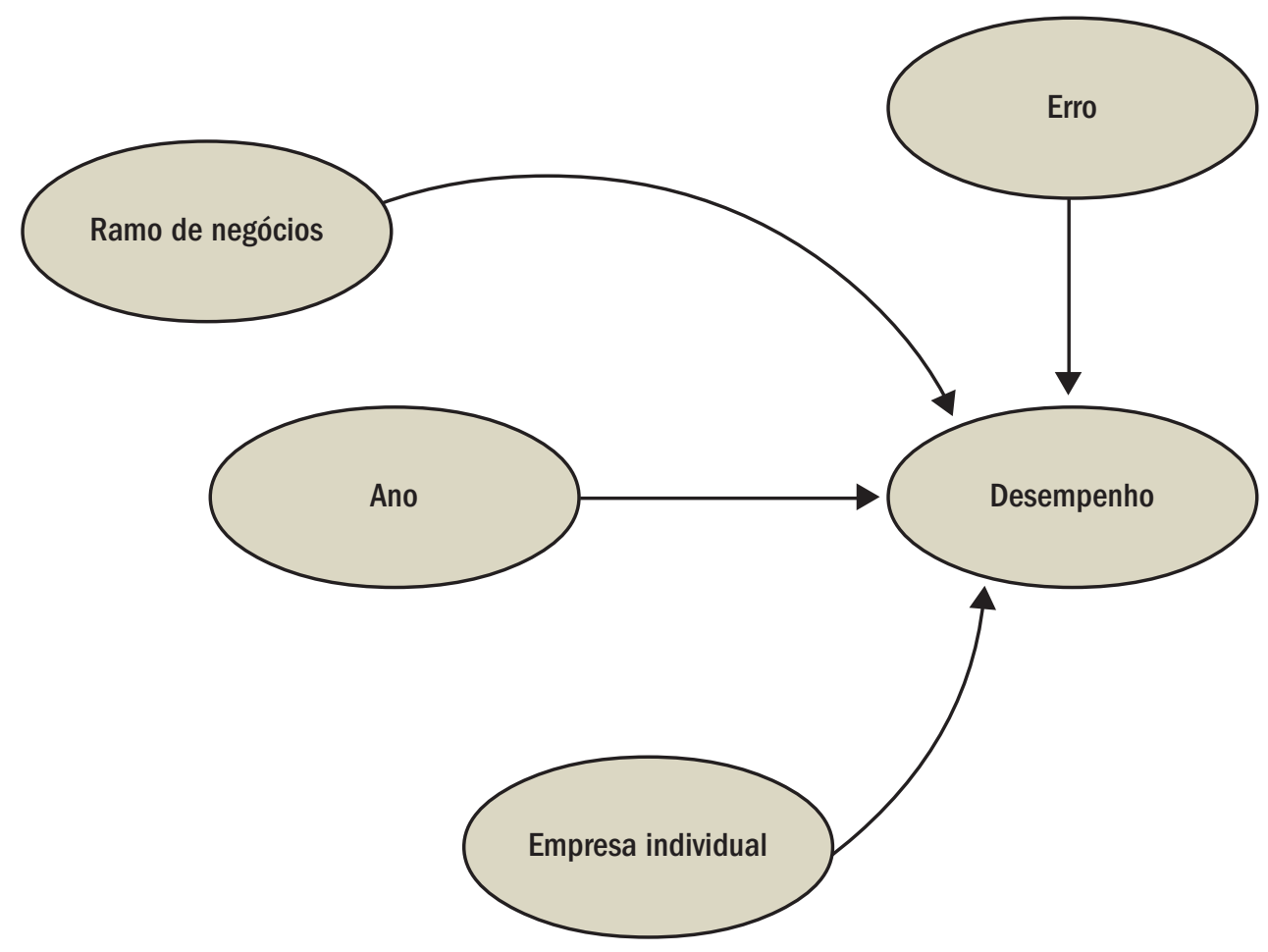


desvantagem competitiva (componente). A vantagem competitiva resultante seria, portanto, o resultado final de todas as influências idiossincráticas da empresa sobre o seu resultado, positivas, negativas e efeitos das interações.

\section{Temporária ou sustentável?}

A proposta deste estudo também resolve a questão da temporalidade da vantagem competitiva. A vantagem competitiva sempre estaria associada a um determinado intervalo de tempo. Não se poderia falar que uma determinada empresa tem ou não tem vantagem competitiva. A própria definição da vantagem competitiva implica uma definição do intervalo de tempo, já que ela seria a resultante de todos os fatores idiossincráticos da empresa, que se mantiveram constantes durante o intervalo de tempo considerado. Um lance de sorte, por exemplo, que tenha influenciado o resultado de uma empresa em um período (ano, trimestre ou mês específico) não seria considerado como vantagem competitiva, mesmo que este impacto fosse relevante. Essa proposta tem relação com a questão da sustentabilidade. Estamos, portanto, definindo, e posteriormente medindo, uma vantagem competitiva resultante, sustentada durante todo o intervalo especificado. O intervalo de tempo pode ser variado, para menos ou para mais, $\mathrm{e}$ faz parte da definição. Intervalos menores seriam capazes de captar efeitos mais transientes, e intervalos maiores tenderiam a captar apenas diferenças mais persistentes. Vejamos a seguir como essa proposta foi operacionalizada.

\section{METODOLOGIA}

\section{Definição do modelo operacional}

O modelo original usado nos estudos de componentes de variância expressa o desempenho em função das diferentes origens da variação dos resultados. A parte da variação não explicada por qualquer um dos efeitos é agrupada em um termo de erro. Assim, a expressão matemática do modelo é:

$$
r_{i, k, t}=\mu+\gamma_{t}+\alpha_{i}+\phi_{k}+\varepsilon_{i, k, t}
$$

A variável dependente $r_{i, k, t}$ representa o indicador selecionado de desempenho da empresa estudada. No caso deste estudo, o indicador é o ROA (Retorno sobre Ativos) de uma empresa específica $k$, em um ramo de negócios específico $i$, no ano $t$. O termo $\mu$ é a média geral de todas as observações consideradas e os demais termos são as influências positivas e negativas em relação à média de cada fator considerado. Assim, o termo $\gamma_{t}$ representa a influência do ano $t$ para o resultado de todas as empresas naquele ano. Se, por exemplo, no ano de 1998, todas as empresas da amostra tiveram seus resultados influenciados positivamente por um conjunto de fatores macroeconômicos, o valor de $\gamma_{1998}$ será adicionado à média geral para compor o valor final da variável dependente $r$ para todas as empresas naquele ano.

O termo $\alpha_{i}$ representa a influência do ramo de negócios $i$, ao qual a empresa pertence. Assim, se o fato de pertencer a um setor como o farmacêutico afeta positivamente todas as empresas que pertencem a esse setor, o valor de $\alpha_{\text {farmacêutico }}$ será um valor positivo e será adicionado à média geral para compor o valor previsto do desempenho de cada empresa. O termo $\phi_{k}$ representa os fatores específicos a uma empresa. Se uma empresa apresenta valores consistentemente positivos e elevados em todas as observações realizadas, e essa persistência de resultados é associada apenas à empresa específica, essa empresa tem algo especial relativo apenas a ela. Esse termo capta essa influência e a separa da influência do setor industrial, do ano $\varepsilon$, até mesmo, da influência de fatores aleatórios, não controlados, incluídos no termo de erro. Daí resulta a proposta deste trabalho, em defender que esse termo é uma estimativa da vantagem (ou desvantagem, se negativo) competitiva dessa empresa em relação à média geral das empresas analisadas. Finalmente, o termo $\varepsilon_{i, k, t}$ capta todos as fontes de variação não captadas pelos termos anteriores.

Esse modelo básico pode ser ampliado e modificado de várias formas. Uma possibilidade é incluir termos de interação entre os fatores analisados. Rumelt (1991) incluiu um termo de interação entre ano e ramo de negócios. O termo de interação capta variações específicas à combinação dos dois fatores em interação. Assim, se um determinado ramo de negócios é especialmente beneficiado ou prejudicado em um ano específico, este termo capta esse tipo de variação. A inclusão deste termo permitiu a Rumelt (1991) separar a influência do ramo de negócios em uma influência fixa, o termo original $\alpha_{i}$ do modelo original (1), e uma influência transiente representada pela interação entre o fator ramo de negócios e o ano, o termo $\delta_{i t}$ no modelo modificado (2).

$$
r_{i, k, t}=\mu+\gamma_{t}+\alpha_{i}+\delta_{i t}+\phi_{k}+\varepsilon_{i, k, t}
$$

Outras possibilidades de modificação são as inclusões de outros termos, como o efeito corporação, que 
representaria o efeito nos resultados da empresa individual pelo fato de pertencer a uma corporação maior. Os dados deste estudo não permitem essa análise por não trazerem detalhes quanto à corporação. O tema "efeitos corporativos" foi amplamente estudado por vários autores e uma revisão ampla pode ser vista em Bowman e Helfat (2001). Khanna e Rivkin (2001) usaram um modelo semelhante, incluindo o efeito de pertencer a um grupo econômico em países emergentes. É possível também incluir um efeito país, incluindo variáveis dummy para cada país individual (Brito e Vasconcelos, 2003).

Uma alternativa a esse tipo de modelo é a inclusão de termos auto-regressivos, tal como feito por Waring (1996) e McGahan e Porter $(1997,1999)$. O uso de termos autoregressivos é mais interessante quando se deseja analisar a persistência de determinado efeito, como é o caso de Waring (1996) e McGahan e Porter (1999). No caso em questão, a sua inclusão poderia dificultar a interpretação dos coeficientes que foram tomados como uma medida da vantagem competitiva, já que parte da variação ficaria com o termo auto-regressivo e não conseguiríamos isolar o efeito empresa que buscamos. Outro aspecto a considerar é que a introdução de um termo auto-regressivo não garante um melhor poder explicativo ao modelo. No caso de McGahan e Porter (1997), o modelo com termo auto-regressivo foi capaz de explicar $51,60 \%$ da variância total, enquanto os mesmos dados analisados com o modelo de Rumelt (1991), sem termo auto-regressivo e mais similar ao modelo deste trabalho, foi capaz de explicar $54,23 \%$ da variância total.

Os trabalhos realizados no campo de estratégia com o uso do tipo de modelo acima descrito concentraram-se na estimativa dos percentuais de variância associados a cada um desses fatores. Nesses trabalhos, o objetivo não foi determinar os valores específicos de cada termo, mas sim avaliar quanto a variação associada a cada classe de termo contribuía para a variância total da variável dependente. Nessa abordagem, os efeitos são tratados como efeitos aleatórios.

Este trabalho tem uma abordagem diferente. O objetivo não é determinar a composição da variância, repetindo o que foi feito em trabalhos anteriores, mas sim estimar o valor específico de cada fator nos seus diversos níveis. Estamos, pois, interessados em estimar os valores específicos de $\phi_{k}$ para cada uma das empresas na amostra. Esses valores de $\phi_{k}$ podem, então, ser interpretados como uma estimativa da vantagem competitiva dessa empresa durante o período de análise considerado.
Para isso, o modelo (1) foi desenvolvido como uma regressão múltipla com variáveis dummy. Essa abordagem é equivalente à análise de variância com efeitos fixos (Neter et al., 1996, p. 832-836). A forma geral da regressão é dada pela equação abaixo:

$$
\hat{r}_{i, t, k}=\mu_{. .}+\sum_{t=1}^{T} \gamma_{t} \cdot A_{t}+\sum_{i=1}^{I} \alpha_{i} \cdot I_{i}+\sum_{k=1}^{K} \phi_{k} \cdot E_{k}
$$

em que:

$\hat{r}_{i, t, k}:$ são os valores do retorno sobre ativos (ROA);

$\mu_{.}$: é a média geral dos valores de retorno sobre ativos;

$A_{t}$ : são as variáveis dummy referentes ao ano;

$\gamma_{t}$ : são os coeficientes obtidos na regressão para as variáveis dummy ano;

$I_{i}$ : são as variáveis dummy referentes ao ramo de negócios;

$\alpha_{i}$ : são os coeficientes para as variáveis dummy ramo de negócios;

$E_{k}$ : são as variáveis dummy referentes a cada uma das empresas consideradas;

$\phi_{k}$ : são os coeficientes para as variáveis dummy de cada empresa.

Para explicitar os coeficientes como efeitos em relação à média geral, foi usado o método de codificação por efeitos, que atribui o valor -1 ao caso base (Cohen e Cohen, 1983, p. 200-204).

\section{Dados utilizados}

Os dados foram extraídos da base de dados Compustat Global. Neste estudo, foram consideradas apenas empresas que atuam nos ramos de negócios classificados como manufatura pela classificação SIC (Standard Industry Classification). Além disso, apenas empresas com receitas líquidas e ativos totais superiores a US\$ 10 milhões foram consideradas. Dois grupos de empresas foram analisados. O primeiro grupo refere-se aos países pertencentes ao NAFTA (México, Estados Unidos e Canadá); o segundo refere-se a países europeus (Suécia, Portugal, Noruega, Holanda, Itália, Irlanda, Grã-Bretanha, França, Finlândia, Espanha, Dinamarca, Alemanha e Áustria). As duas amostras representam um total de 13.259 observações sobre 2.805 empresas, compreendendo dados de 1997 a 2001. Características descritivas desses dois grupos podem ser vistas na tabela 1 .

A medição da vantagem competitiva tem de começar sempre com a medição do desempenho ou do sucesso empresarial. O desempenho tem múltiplas dimensões e não há consenso sobre o modo como medi-lo (Barney, 2002, p. 28; Meyer, 2002, p. 19-49; Chakravarthy, 1986; 
Kaplan e Norton, 1996, p. 24). Diversos trabalhos que analisaram os componentes de variância do desempenho da empresa exploraram diferentes formas de medição, chegando, na maioria dos casos, a conclusões similares (Montgomery e Wernerfelt, 1988; Powell, 1996; McGahan, 1999; Hawawini, Subramanian e Verdin, 2003).

A proposta deste trabalho permite medir a vantagem competitiva em qualquer dimensão escolhida para o desempenho. Neste trabalho, escolhemos a variável "retorno sobre ativos", seguindo a tradição dominante nesta linha de pesquisa (para uma análise comparativa de diferentes formas de medição ver Hawawini, Subramanian e Verdin, 2003).

\section{RESULTADOS}

O modelo de regressão, aplicado às duas amostras mencionadas anteriormente, usou 1.712 variáveis dummy para o grupo de empresas da amostra NAFTA e 1.508 variáveis dummy para o grupo de empresas européias. A regressão mostrou-se altamente significativa, com valor de $R^{2}$ acima de 0,50 para ambos os casos e p-values menores do que 0,001 para o teste $F$, como mostra a tabela 2 .

Coeficientes correspondentes às empresas individuais foram calculados para as empresas das duas amostras. O valor desses coeficientes será tomado como estimativa da vantagem competitiva dessas empresas. Dado o elevado número de empresas envolvidas nas duas amostras,

Tabela 1 - Dados das amostras analisadas.

\begin{tabular}{|l|c|c|}
\hline \multicolumn{1}{|c|}{ VARIÁVEIS } & NAFTA & EUROPA \\
\hline Empresas & 1.502 & 1.303 \\
\hline SICs & 208 & 203 \\
\hline Anos & 5 & 5 \\
\hline Países & 3 & 13 \\
\hline Total de observações & 7.120 & 6.139 \\
\hline Média ROA & $1,49 \%$ & $3,76 \%$ \\
\hline Desvio padrão & $20,08 \%$ & $10,54 \%$ \\
\hline
\end{tabular}

Tabela 2 - Resultados das regressões para as duas amostras.

\begin{tabular}{|l|c|c|}
\hline \multicolumn{1}{|c|}{ VARIÁVEIS } & NAFTA & EUROPA \\
\hline Número de variáveis dummy & 1.712 & 1.508 \\
\hline R quadrado da regressão & 0.530 & 0.520 \\
\hline R quadrado ajustado & 0.405 & 0.390 \\
\hline$p$-value (teste F) & 0.000 & 0.000 \\
\hline
\end{tabular}

a análise deve ser feita com técnicas de estatística descritiva. Coeficientes positivos indicam que efeitos específicos à empresa individual contribuem para o desempenho de forma positiva em relação à média geral. Coeficientes negativos indicam o contrário - os efeitos específicos à empresa individual estão reduzindo o valor de seu desempenho em relação à média. As distribuições desses coeficientes, apresentadas sob forma de histograma na figura 2 e na figura 3, mostram que esses coeficientes apresentam uma distribuição aproximadamente simétrica em torno da média zero.

A metodologia de regressão permite o uso de um teste estatístico para verificar se o coeficiente de uma empresa específica é significativamente diferente de zero. Esse teste seria a comprovação estatística da existência da vantagem ou da desvantagem competitiva. Essa possibilidade é especialmente importante por permitir a mensuração da vantagem competitiva e, também, a avaliação da sua freqüência, respondendo a questões empíricas importantes, como 1) quantas empresas têm vantagem competitiva? e 2) quantas empresas têm desvantagem competitiva?

A aplicação de um teste $t$ unilateral com nível de significância de 5\% nos permitiu responder a essas duas questões como segue: 1) 1,0\% das empresas do grupo NAFTA e 12,5\% das empresas européias têm vantagem competitiva estatisticamente demonstrável; 2) 11,8\% das empresas do grupo NAFTA e 10,0\% das empresas européias têm desvantagem competitiva estatisticamente demonstrável.

Esses resultados indicam que tanto a vantagem competitiva quanto a desvantagem são fenômenos freqüentes, correspondendo a mais de $20 \%$ da amostra de empresas testadas, contrariando o que afirma Powell (2003), que se refere ao desempenho excepcional como um fato incomum, quase um outlier.

\section{DISCUSSÃO E CONCLUSÕES}

Ao definir estratégia como uma teoria de como uma empresa pode competir com sucesso, a conquista da vantagem competitiva é o objetivo último da estratégia (Barney, 2002). Embora nem todas as teorias de estratégia estejam prioritariamente focalizadas na procura ou maximização da lucratividade (Whittington, 2001), a maioria dos autores nesse campo reconhece que as empresas privadas se orientam pela procura da lucratividade crescente, e que as teorias de estratégia tendem a ser teorias de maximização de lucratividade. 
Os fenômenos de lucratividade persistentemente diferentes da média de mercado são, dessa maneira, naturais e até desejáveis, testemunhando o sucesso dos modelos teóricos da disciplina (Barney, 1986, 2001; Porter, $1979,1980,1985)$. Por contraste, a microeconomia tende a ser um campo acadêmico cujo foco recai sobre a explicação da homogeneidade, da perfeição dos mercados, dos fluxos de informação instantâneos e da fluidez dos modelos matemáticos. Em tal disciplina, tudo se estrutura para mostrar como os mercados convergem automaticamente para o equilíbrio, e fenômenos como a vantagem competitiva não passam de perturbações transitórias (Rumelt, 1991).

Neste trabalho, procuramos demonstrar que o conceito de vantagem competitiva não tem uma operacionalização detalhada e compartilhada pelos pesquisadores do tema, sendo motivo de crescente polêmica no meio acadêmico (Barney, 1991; Besanko et al., 2004). Nesse contexto, o presente trabalho oferece uma contribuição ao debate ao propor uma definição constitutiva e operacional da vantagem competitiva, coerente com a maioria dos desenvolvimentos teóricos na área, inspirando-se na série de trabalhos de componentes de variância do desempenho. A essência dessa definição consiste em ver a vantagem competitiva como a resultante de todos os fatores específicos da empresa em particular durante um período específico.

Se o fato de pertencer a um determinado setor industrial não pode ser considerado uma fonte de vantagem competitiva, uma posição de mercado específica privilegiada dentro da indústria, ocupada somente por uma empresa em particular, que proporcione um resultado superior, será uma das contribuições (uma vantagem competitiva componente), entre outras, para a vantagem competitiva resultante final da empresa.

Cool, Costa e Dierickx (2002) argumentam que a vantagem competitiva pode ser obtida tanto por uma posição privilegiada na indústria, posição essa que não pode ser replicada por outros (Caves, 1984), como por recursos obtidos em mercados de fatores imperfeitos (Barney, 1986a), não completamente móveis (Peteraf, 1993), protegidos da imitação (Rumelt, 1984; Dierickx e Cool, 1989; Reed e DeFillippi, 1990), e não substituíveis - os recursos estrategicamente equivalentes de Barney (1991). A proposta de definição apresentada neste estudo trata essas fontes como geradoras de vantagens competitivas componentes, oferecendo um mecanismo de integração das diferentes abordagens.

Figura 2 - Histograma dos coeficientes da regressão dos países pertencentes ao NAFTA.

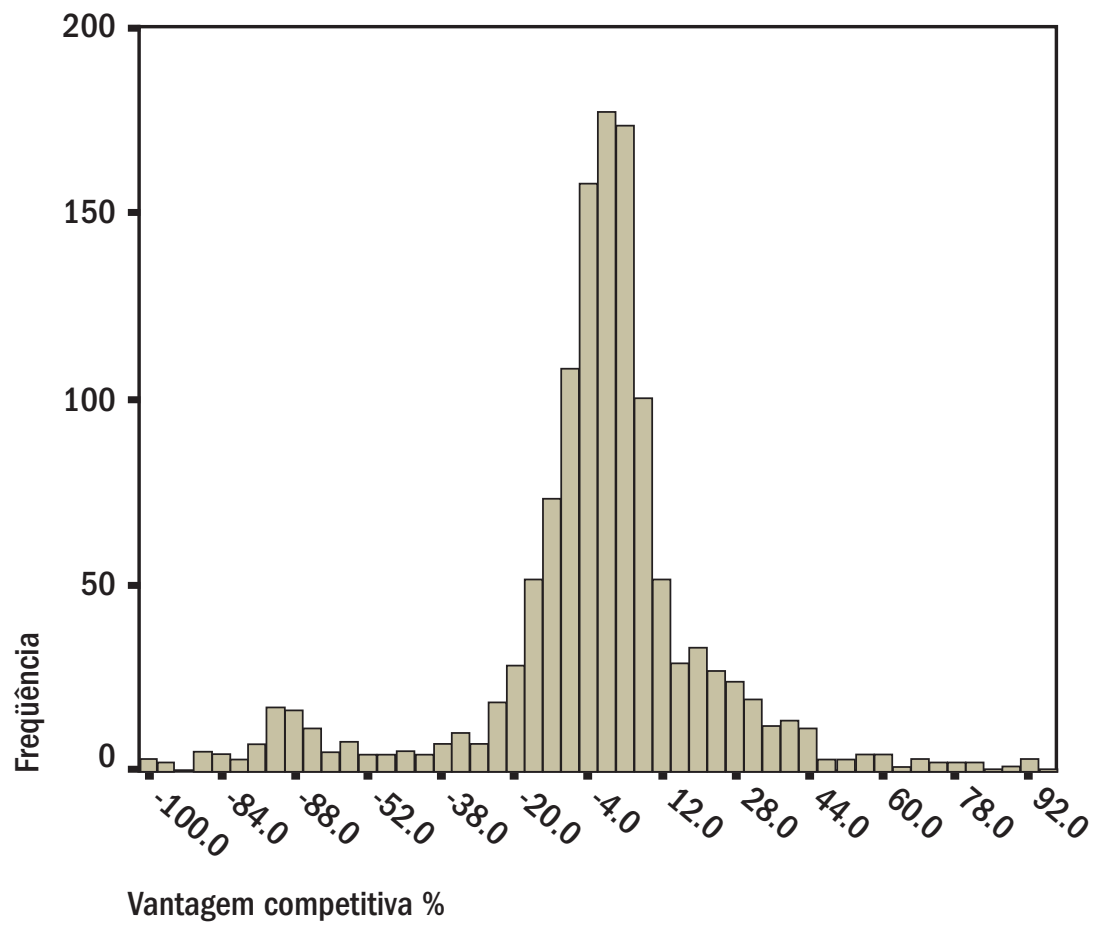


A conceituação aqui proposta esclarece também a questão temporal da definição por meio da noção de vantagem competitiva média. O que medimos é a vantagem competitiva média, sustentada durante todo o período especificado. O período faz parte da definição. Essa proposta qualifica a proposição de Jacobsen (1988) e Porter (1985), que se referem a um tempo suficientemente longo. Intervalos de tempo mais longos tenderão a reduzir o que chamamos de vantagem competitiva, pois o diferencial de desempenho atribuível à empresa deveria estar presente durante todo o intervalo determinado.

Assim como o desempenho é multidimensional e pode ser avaliado em várias dimensões e unidades de medição, a vantagem competitiva pode ser expressa nas mesmas dimensões e unidades (March e Sutton, 1997; Venkatraman e Ramanujam, 1986). O conceito aqui proposto também contempla esse aspecto, permitindo diferentes operacionalizações com os vários tipos de indicadores de desempenho. Lippmann e Rumelt (2003a) defendem a posição de que a abordagem microeconômica de lucro econômico, que foi usada no início da RBV para definir vantagem competitiva, tem falhas e inconsistências graves e deveria ser substituída por uma abordagem muito mais simples, que ignore o "custo" do uso alternativo dos recursos, e se baseie em algo muito próximo de um simples fluxo de caixa. A proposta de definição de vantagem competitiva aqui defendida ajusta-se muito bem a essa colocação, já que várias das operacionalizações possíveis podem usar medidas diretamente associadas ao fluxo de caixa ou os pagamentos recebidos pela empresa.

Uma forma alternativa de definir um desempenho superior é adotar um modelo de barganha em que se considera o valor final para o comprador como a medida principal. A vantagem competitiva determinaria então a diferença entre esse valor e o custo. O preço seria determinado por um mecanismo de barganha entre o vendedor e o comprador, definindo, então, qual parte do valor seria apropriada por um ou por outro (Hoopes, Madsen e Walker, 2003). Vários livros-texto de estratégia usam essa estrutura para definir a vantagem competitiva (Besanko et al., 2004, p. 367-373; Walker, 2004, cap. 2). As dificuldades de medição são óbvias. A apropriação do valor é tratada separadamente da criação deste. Lippman e Rumelt (2003b) propõem o uso da teoria de jogos cooperativos em conjunto com a RBV para buscar a solução dessa questão. Coff (1999) adota uma perspectiva ainda mais ampla, considerando todo o valor gerado por uma empresa como o resultado da vantagem competitiva. Nessa abordagem, o processo de barganha por salários

Figura 3 - Histograma dos coeficientes da regressão dos países europeus.

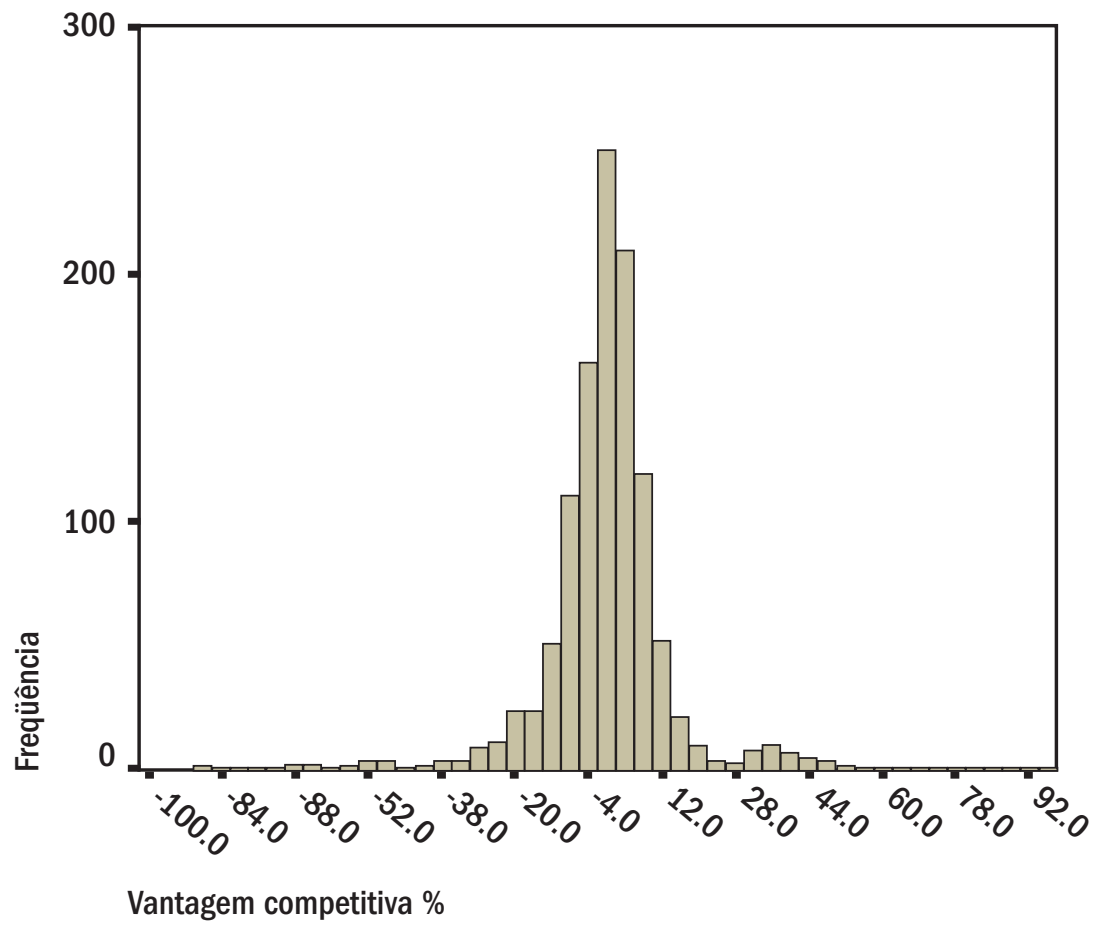

$60 \cdot \mathrm{ORAE} \cdot \mathrm{VOL} 44 \cdot \mathrm{N}^{\circ} 2$ 
entre a empresa e seus empregados, por exemplo, poderia dividir esse valor de forma a pagar salários muito altos e a empresa não apresentar lucro devido a isso, mesmo que ainda tivesse uma vantagem competitiva.

$\mathrm{Na}$ definição aqui proposta, a capacidade de apropriação do valor criado pela empresa é tratada como um dos componentes da vantagem competitiva, se essa capacidade for algo específico à empresa individual. Se a capacidade de se apropriar do valor for uma característica de um grupo de empresas, como um ramo de negócios, por exemplo, as conseqüências dessa capacidade não estariam incluídas na definição, já que afetariam todos os participantes do ramo de negócios e não seriam fonte de diferenciação de desempenho entre eles.

O presente estudo também ilustrou uma forma de operacionalizar a definição de vantagem competitiva, e essa operacionalização traz vantagens em relação à simples comparação direta do desempenho. Em primeiro lugar, ela isola a influência dos fatores específicos da empresa, descontando a influência do ramo de negócios, do ano específico de observação, fatores aleatórios e quaisquer outros que possam ser incluídos em um modelo mais aprimorado. Em segundo lugar, ela permite a comparação entre ramos de negócios diferentes. A abordagem tradicional, de primeiro segmentar os resultados tomando empresas do mesmo ramo de negócios e só depois analisar as diferenças entre empresas, tem limitações. O número de observações envolvido pode ser muito pequeno para conclusões mais abrangentes, a natureza das relações entre as empresas não é restrita a um ramo de negócios e as relações não são apenas competitivas, mas também envolvem elementos de cooperação (Brandenburger e Nalebuff, 1996). A abordagem aqui proposta trata a população de empresas como um conjunto integrado. Em terceiro lugar, a abordagem permite um teste estatístico com maior poder de definição. O modelo proposto para esta operacionalização deve ser tomado apenas como uma ilustração de outras operacionalizações possíveis. Outros termos que representem o efeito da corporação, do país, de um eventual cluster ao qual pertença a empresa, podem ser incluídos e pesquisados. Termos auto-regressivos podem ser explorados (McGahan e Porter, 1997). Modelos multiníveis (hierárquicos) podem ser usados para análises mais amplas (Raudenbusch e Bryk, 2002).

A literatura está repleta de análises que avaliam o efeito de determinado recurso ou posição de mercado como vantagem competitiva, ou o seu efeito no desempenho das empresas. March e Sutton (1997) fazem uma crítica metodológica contundente a esse tipo de estudo, baseada na impossibilidade de controlar todos os outros efeitos de variáveis intervenientes no desempenho. Na vida real, as empresas estão sujeitas a uma multiplicidade de influências concomitantes. O seu desempenho é o resultado combinado de todas elas. Mesmo quando isolamos os fatores específicos da empresa individual durante um período específico, estamos medindo uma resultante. O conceito aqui proposto de vantagem competitiva é, nesse aspecto, diferente de muitos tratamentos dados a ele em estudos sobre estratégia. O que estamos medindo é a vantagem competitiva resultante, que permite conceber, de forma análoga, as vantagens competitivas componentes. Definindo claramente como medir a resultante, é possível desenvolver estudos e modelos que possam estimar seus componentes, mas é difícil conceber o caminho inverso, já que nunca poderemos saber quantas componentes faltam e a influência que elas podem ter nas que estamos analisando, tal como colocam March e Sutton (1997).

\section{REFERÊNCIAS BIBLIOGRÁFICAS}

AAKER, D. A. How to select a business strategy. California Management Review, v. 26, n. 3, p. 167-175, 1984.

ALLEN, M. G. Strategic planning with a competitive focus. The McKinsey Quarterly, Autuum, n. 3, p. 2-13, 1978.

ANDREWS, K. R. The concept of corporate strategy. Homewood: Dow Jones-Irwin, 1971.

ANSOFF, H. I. Corporate strategy. Harmondsworth: Penguin, 1965.

ANSOFF, H. I. Strategic management. New York: Wiley, 1979.

AREND, R. J. Revisiting the logical and research considerations of competitive advantage. Strategic Management Journal, v. 24, n. 3, p. 279284,2003

BARNEY, J. Strategic factor markets: expectations, luck and business strategy. Management Science, v. 32, n. 10, p. 1231-1241, 1986a.

BARNEY, J. Organizational culture: can it be a source of sustained competitive advantage? Academy of Management Review, v. 11, n. 3, p. 656$665,1986 b$.

BARNEY, J. Firm resources and sustained competitive advantage. Journal of Management, v. 17, n. 1, p. 99-120, 1991.

BARNEY, J. Resource-based theories of competitive advantage: a ten year retrospective on the resource-based view. Journal of Management, v. 27, n. 6, p. 643-650, 2001. 
BARNEY, J. Gaining and sustaining competitive advantage. Upper Saddle River: Prentice-Hall, 2002.

BESANKO, D. et al. Economics of strategy. New York: Wiley, 2004.

BOWMAN, E. H.; HELFAT, C. E. Does corporate strategy matter? Strategic Management Journal, v. 21, n. 1, p. 1-24, 2001.

BRANDENBURGER, A. M.; NALEBUFF, B. J. Co-opetition. New York: Doubleday, 1996.

BRITO, L. A. L.; VASCONCELOS, F. C. How much does country matter? In: ENCONTRO ANUAL DA ASSOCIAC̄̃̃O NACIONAL DOS PROGRAMAS DE PÓS-GRADUAÇÃO EM ADMINISTRAÇÃO, 27º, 2003, Atibaia. Anais... Atibaia: Anpad, 2003.

CAVES, R. E. Economic analysis and the quest for competitive advantage. The American Economic Review, v. 74, n. 2, p. 127-132, 1984.

CHAKRAVARTHY, B. S. Measuring strategic performance. Strategic Management Journal, v. 7, n. 5, p. 437-458, 1986.

COFF, R. W. When competitive advantage doesn't lead to performance: the resource-based view and stakeholder bargaining power. Organization Science, v. 10, n. 2, p. 119-133, 1999.

COHEN, J.; COHEN, P. Applied multiple regression/correlation analysis for the behavioral sciences. Hillsdale: Lawrence Earlbaum Associates, 1983.

COOL, K.; COSTA, L. A.; DIERICKX, I. Constructing competitive advantage. In: PETTIGREW, A.; THOMAS, H.; WHITTINGTON, R. Handbook of strategic management. London: Sage, 2002.

DURAND, R. Competitive advantages exist: a critique of Powell. Strategic Management Journal, v. 23, n. 9, p. 867-872, 2002.

GHEMAWAT, P. Sustainable advantage. Harvard Business Review, v. 64, n. 5, p. $53 .-58,1986$.

GLUCK, F. W.; KAUFMAN, S. P.; WALLECK, A. S. Strategic management for competitive advantage. Harvard Business Review, v. 58, n. 4, p. 154161,1980

HAWAWINI, G.; SUBRAMANIAN, V.; VERDIN, P. Is performance driven by industry - or firm -specific factors? A new look at the evidence. Strategic Management Journal, v. 24, n. 1, p. 1-16, 2003.

HAYES, R. H.; WHEELWRIGHT, S. C. Restoring our compettive edge. New York: Wiley, 1984.

HITT, M. A.; IRELAND, R. D.; HOSKISSON, R. E. Strategic management. Cincinnati: South Western College, 1999.

HOOPES, D.; MADSEN, T.; WALKER, G. Guest editor's introduction to the special issue: why is there a resource based view? Toward a theory of competitive heterogeneity. Strategic Management Journal, v. 24, n. 10, p. 889-902, 2003.

JACOBSEN, R. The persistence of abnormal returns. Strategic Management Journal, v. 9, n. 5, p. 415-430, 1988.
KAPLAN, R. S.; NORTON, D. P. The balanced scorecard: translating strategy into action. Boston: Harvard Business School, 1996.

KHANNA, T.; RIVKIN, J. W. Estimating the performance effects of business groups in emerging markets. Strategic Management Journal, v. 22, n. 1, p. 45-74, 2001.

LEVITAS, E.; CHI, T. Rethinking rouse and daellenbach's rethinking: isolating vs. testing for sources of sustainable competitive advantage. Strategic Management Journal, v. 23, n. 10, p. 957-962, 2002.

LIPPMAN, S.; RUMELT, R. The payments perspective: micro-foundations of resource analysis. Strategic Management Journal, v. 24, n. 10, p. $903-$ 927, 2003a.

LIPPMAN, S.; RUMELT, R. A bargaining perspective on resource advantage. Strategic Management Journal, v. 24, n. 11, p. 1069-1086, 2003 b.

MARCH, J. G.; SUTTON, R. I. Organizational performance as a dependent variable. Organization Science, v. 8, n. 6, p. 698-706, 1997.

McGAHAN, A. M. The performance of US corporations: 1981-1994. The Journal of Industrial Economics, v. XLVII, n. 4, p. 373-398, 1999.

McGAHAN, A. M.; PORTER, M. E. How much does industry matter, really? Strategic Management Journal, v. 18, n. 1, p. 15-30, 1997.

McGAHAN, A. M.; PORTER, M. E. The persistence of shocks to profitability? The Review of Economics and Statistics, v. 81, n. 1, p. 143-153, 1999.

MEYER, M. W. Rethinking performance measurement. Cambridge: Cambridge University, 2002.

MONTGOMERY, C. A.; WERNERFELT, B. Diversification, ricardian rents, and Tobin's q. The Rand Journal of Economics, v. 19, n. 4, p. 623-610, 1988 .

MORRISON, J. R.; LEE, J. G. The anatomy of strategic thinking. The McKinsey Quarterly, Autumn, n. 3, p. 2-9, 1979.

NETER, J. et al. Applied linear regression models. Chicago: Irwin, 1996.

OHMAEL, K. Effective strategies for competitive success. The McKinsey Quarterly, Winter, n. 4, p. 50-59, 1978.

PETERAF, M. The cornerstones of competitive advantage - a resourcebased view. Strategic Management Journal, v. 12, n. 3, p. 95-117, 1993.

PORTER, M. Competitive strategy: techniques for analyzing industries and competitors. New York: Free Press, 1980.

PORTER, M. Competitive advantage: creating and sustaining superior performance. New York: Free Press, 1985.

PORTER, M. E. The structure within industries and companies' performance. The Review of Economics and Statistics, v. 61, n. 2, p. 214-227, 1979. 


\section{FLÁVIO CARVALHO DE VASCONCELOS · LUIZ ARTUR LEDUR BRITO}

POWELL, T. C. How much does industry matter? An alternative empirical test. Strategic Management Journal, v. 17, n. 4, p. 323-312, 1996.

POWELL, T. C. Competitive advantage: logical and philosophical considerations. Strategic Management Journal, v. 22, n. 9, p. 875-888, 2001

POWELL, T. C. The philosophy of strategy. Strategic Management Journal, v. 23, n. 9, p. 873-880, 2002.

POWELL, T. C. Varieties of competitive parity. Strategic Management Journal, v. 24, n. 1, p. 61-86, 2003.

PRAHALAD, C. K.; HAMEL, G. The core competence of the corporation. Harvard Business Review, v. 68, n. 3, p. 79-92, 1990.

PRIEM, R. L.; BUTLER, J. E. Is the resource based "view" a useful perspective for strategic management research? Academy of Management Review, v. 26, n. 1, p. 22-40, 2001

RAUDENBUSCH, S. W.; BRYRK, A. S. Hierarchical linear models: applications and data analysis methods. Thousand Oaks: Sage, 2002.

REED, R.; DeFILLIPPI, R. J. Causal ambiguity, barriers to imitation, and sustainable competitive advantage. Academy of Management Review, v. 15, n. 1 , p. $88-102,1990$.

ROQUEBERT, J. A.; PHILliPS, R. L.; WeSTFALL, P. A. Markets vs. management: what drives profitability? Strategic Management Journal, v. 17, n. 8 , p. $653-664,1996$.

ROTHSCHILD, W. E. Surprise and the competitive advantage. The Journal of Business Strategy, v. 4, n. 3, p. 10-18, 1984a.

ROTHSCHILD, W. E. How to gain (and maintain) the competitive advantage in business. New York: McGraw-Hill, 1984b.

ROUSE, M. J.; DAELLENBACH, U. S. Rethinking research methods for the resource-based perspective: isolating sources of sustainable competitive advantage. Strategic Management Journal, v. 20, n. 5, p. 487-494, 1999.
ROUSE, M. J.; DAELLENBACH, U. S. More thinking on research methods for the resource-based perspective. Strategic Management Journal, v. 23, n. 10, p. 963-967, 2002.

RUMELT, R. How much does industry matter? Strategic Management Journal, v. 12, n. 3, p. 167-119, 1991.

RUMELT, R. P.; SCHENDEL, D.; TEECE, D. J. Strategic management and economics. Strategic Management Journal, v. 12, Special Issue, p. 5-29, 1991

RUMELT, R. P.; SCHENDEL, D.; TEECE, D. J. Fundamental issues in strategy: a research agenda. Boston: Harvard Business School, 1994.

SCHMALENSEE, R. Do markets differ much? The American Economic Review, v. 75, n.. 3, p. 341-351, 1985

SOUTH, S. E. Competitive advantage: the cornerstone of strategic thinking. The Journal of Business Strategy, v. 1, n. 4, p. 15-25, 1981.

SPENCE, A. M. Industrial organization and competitive advantage in multinational industries. The American Economic Review, v. 74, n. 2, p. 356360, 1984.

VENKATRAMAN, N.; RAMANUJAM, V. Measurement of business performance in strategy research: a comparison of approaches. The Academy of Management Review, v. 11, n. 4, p. 801-814, 1986.

WALKER, G. Modern competitive strategy. Boston: McGraw-Hill Irwin, 2004

WARING, G. Industry differences in the persistence of firm-specific returns, The American Economic Review, v. 86, n. 5, p. 1253-1265, 1996.

WERNERFELT, B. A resource-based view of the firm. Strategic Management Journal, v. 5, n. 2, p. 171-180, 1984

WHEELWRIGHT, S. C. Japan - where operations are really strategic Harvard Business Review, v. 59, n. 4, p. 67-74, 1981

WHITTINGTON, R. What is strategy - and does it matter? London: Thomson Learning, 2001.

Artigo recebido em 29.07.2003. Aprovado em 04.03.2004.

\section{Flávio Carvalho de Vasconcelos}

Professor do Departamento de Administração Geral e Recursos Humanos da FGV-EAESP. Doutor em Administração de Empresas pela HEC-Ecole des Hautes Etudes Commerciales, Paris. Interesses de pesquisa em estratégia e organizações. E-mail: fvasconcelos@fgvsp.br

Endereço: Av. Nove de Julho, 2029, 9oandar, Bela Vista - São Paulo - SP, 01313-902.

\section{Luiz Artur Ledur Brito}

Professor do Departamento de Produção e Operações Industriais da FGV-EAESP. Doutorando em Administração de Empresas pela FGV-EAESP. Interesses de pesquisa em estratégia e operações.

E-mail: lbrito@fgvsp.br

Endereço: Av. Nove de Julho, 2029, 10o andar, Bela Vista - São Paulo - SP, 01313-902. 